\title{
Exercise Limitation in Children and Adolescents with Mild-to-Moderate Asthma
}

\author{
Olga Lagiou ${ }^{1,2, *}$ \\ Sotirios Fouzas ${ }^{1,2, *}$ \\ Dimosthenis Lykouras ${ }^{3}$ \\ Xenophon Sinopidis ${ }^{2}$ \\ Ageliki Karatza ${ }^{2}$ \\ Kiriakos Karkoulias ${ }^{3}$ \\ Gabriel Dimitriou ${ }^{2}$ \\ Michael B Anthracopoulos ${ }^{1,2}$ \\ 'Pediatric Respiratory Unit, University of \\ Patras Medical School, Patras, Greece; \\ ${ }^{2}$ Department of Pediatrics, University of \\ Patras Medical School, Patras, Greece; \\ ${ }^{3}$ Department of Respiratory Medicine, \\ University Hospital of Patras, Patras, \\ Greece \\ *These authors contributed equally to \\ this work
}

Background: Children with uncontrolled asthma are less tolerant to exercise due to ventilatory limitation, exercise-induced bronchoconstriction (EIB), or physical deconditioning. The contribution of these factors in children with controlled mild-to-moderate asthma is unknown.

Objective: To explore the underlying mechanisms of reduced exercise capacity in children with controlled mild-to-moderate asthma.

Methods: This was a cross-sectional study of 45 children and adolescents (age 8-18 years) with controlled mild-to-moderate asthma (asthma control test score 21-25) and 61 agematched healthy controls. All participants completed a physical activity questionnaire and performed spirometry and cardiopulmonary exercise testing (CPET; maximal incremental protocol). Spirometric indices and CPET parameters were compared between the two groups. The effect of EIB $\left(\mathrm{FEV}_{1}\right.$ decrease $>10 \%$ post CPET), ventilatory limitation and physical deconditioning on maximum oxygen uptake $\left(\mathrm{VO}_{2}\right.$ peak), was assessed by multivariable linear regression.

Results: $62.2 \%$ of children with asthma and $29.5 \%$ of controls $(P=0.002)$ were categorized as inactive. Reduced exercise capacity ( $\mathrm{VO}_{2}$ peak $<80 \%$ ) was noted in $53.3 \%$ of asthmatics and $16.4 \%$ of controls $(\mathrm{P}<0.001)$. EIB was documented in $11.1 \%$ of participants with asthma. Physical deconditioning was noted in $37.8 \%$ of children with asthma and in $14.8 \%$ of controls $(\mathrm{P}=0.013)$. Physical deconditioning emerged as the only significant determinant of $\dot{\mathrm{VO}}_{2}$ peak, irrespective of asthma diagnosis, body mass index, ventilatory limitation and EIB.

Conclusion: Children with controlled mild-to-moderate asthma are less tolerant to strenuous exercise than their healthy peers. The decreased exercise capacity in this population should mainly be attributed to physical deconditioning, while the contribution of ventilatory limitation and EIB is rather small.

Keywords: asthma, cardiopulmonary exercise testing, children, exercise

\section{Plain Language Summary}

Why was the study done?

Children with severe or uncontrolled asthma experience exercise intolerance due to ventilatory limitation and exercise-induced bronchoconstriction. The contribution of these factors in children with controlled mild-to-moderate asthma is unknown.

What did the researchers do and find?

In this study, 45 children and adolescents with controlled mild-to-moderate asthma and 61 age-matched healthy controls performed cardiopulmonary exercise testing. We found that children with controlled mild-to-moderate asthma were less tolerant to strenuous exercise than their healthy peers. However, their decreased aerobic capacity was mainly attributed to

\footnotetext{
Correspondence: Michael B

Anthracopoulos

Pediatric Respiratory Unit, University of

Patras Medical School, Patras, 26504, Greece

Tel +302610999716

Email manthra@otenet.gr
} 
physical deconditioning, while the contribution of ventilatory limitation and exercise-induced bronchoconstriction was minimal.

What do these results mean?

Our findings suggest that children with mild-to-moderate asthma do not present exercise limitation due to the disease itself and, thus, should be encouraged to participate in physical activities systematically and without constraints.

\section{Introduction}

Asthma represents the most frequent chronic respiratory disorder in childhood. ${ }^{1}$ The disease is characterized by recurrent episodes of reversible airway obstruction that differ in frequency and severity depending on the level of asthma control. ${ }^{2}$ Children with controlled disease do not experience day- or night-time symptoms or limitation of their physical activity; however, exposure to triggering factors, such as viral respiratory infections, allergens, and physical exercise, may unveil asthma symptoms and lead to disease exacerbation. ${ }^{2}$

Although physical activity should be encouraged in children with asthma, ${ }^{3}$ imposed restrictions are not uncommon in this population. ${ }^{4}$ Such attitudes relate primarily to the parental perception that physical exercise may precipitate asthma symptoms ${ }^{5}$ and/or to children's belief that the disease may significantly decrease their physical performance. ${ }^{6}$ However, evidence of a causal relationship between asthma and reduced exercise capacity (ie the amount of physical exertion that an individual can sustain) does not exist, ${ }^{7-11}$ although children with exercise induced bronchoconstriction (EIB) or those with uncontrolled disease tend to be less active than their peers. ${ }^{810-13}$ The few studies in which cardiopulmonary exercise testing (CPET) was employed, suggest that a substantial percentage of children with troublesome disease have reduced exercise capacity simply due to physical deconditioning (ie a decline in physical function of the body as a result of physical inactivity and disuse). ${ }^{13-15}$ The combined effect of EIB, ventilatory limitation (ie a ventilatory output during exercise that closely approaches or matches maximal ventilatory capacity) and physical deconditioning on exercise capacity of pediatric patients with controlled mildmoderate disease, ie on the vast majority of children with asthma, ${ }^{1,2}$ remains largely unknown.

The aim of this study was to explore the underlying mechanisms of reduced exercise capacity in children with controlled mild-to-moderate asthma using CPET. Based on evidence from children with uncontrolled disease, ${ }^{15}$ we hypothesized that ventilatory limitation and EIB may have minimal contribution to the level of exercise capacity in this population.

\section{Materials and Methods Study Design and Population}

This was a cross-sectional study of Caucasian European children aged 8-18 years with mild-to-moderate asthma and age-matched healthy controls. The study was conducted at the CPET laboratory of the Pediatric Respiratory Unit of the University Hospital of Patras, Greece, between June 10 and September 6, 2019.

Participants with asthma were children who: a) attended our Outpatient Asthma Clinics regularly in the last two years, b) had at least one spirometry with $\mathrm{FEV}_{1}<80 \%$ or $\mathrm{FEV}_{1}$ reversibility $\geq 12 \%$ in the previous year, c) were under controller therapy for at least six months in the previous year and, d) were classified as having well controlled, mild-to-moderate asthma (treatment steps 1 and 2), according to Global Initiative for Asthma (GINA) criteria ${ }^{2}$ and the Asthma Control Test (ACT) (ie score $\geq 20$ ). All had interrupted their controller medication for at least four weeks prior to CPET. Healthy controls were recruited from local public schools; ${ }^{16}$ they all had normal spirometry and no asthma diagnosis or prescription of relevant medication within the last two years. All participants had normal echocardiographic examination on the day of enrolment. Exclusion criteria for both groups were: cardiac disease, major disabilities (neurological, muscular, neurodevelopmental), lower respiratory infection in the last month or non-specific respiratory symptoms (including symptoms of allergic rhinitis) in the week prior to enrollment.

The study protocol was approved by the Ethics Committee of the University Hospital of Patras. Study participants and their parents were informed about the purpose of the study. Parental informed written consent and child's informed verbal assent were obtained at enrollment. The study was conducted in accordance with the Declaration of Helsinki.

\section{ACT and Physical Activity Questionnaire}

On the day of study visit, demographic data were collected, and height and weight were measured. The body mass index (BMI) was calculated and assessed ( $z$ scores) according to the International Obesity Task Force norms. ${ }^{17}$ Children with asthma were asked to complete the ageappropriate Greek version of ACT (https://www.asthma 
controltest.com). In addition, all participants completed the Greek version of the International Physical Activity Questionnaire - Short Form (IPAQ-SF) ${ }^{18}$ to estimate the intensity, frequency, and duration of their physical activities. Children with at least 60 minutes of moderate-tovigorous physical activity per day ${ }^{19,20}$ were classified as "active", otherwise they were considered to be "inactive". The ACT and the modified IPAQ-SF were completed in the presence of one of the investigators.

\section{Spirometry}

All participants underwent spirometry using a Micro 5000 spirometer (Medisoft, Sorinnes, Belgium) according to the ERS/ATS guidelines. ${ }^{21}$ Forced expiratory volume at $1 \mathrm{~s}$ $\left(\mathrm{FEV}_{1}\right)$, forced vital capacity (FVC), $\mathrm{FEV}_{1} / \mathrm{FVC}$ ratio, and forced expiratory flow between 25 and $75 \%$ of $\mathrm{FVC}$ $\left(\mathrm{FEF}_{25-75}\right)$ values were assessed according to Global Lung Initiative normative data. ${ }^{22}$ Spirometry was repeated at 5, 10, 15 and 20 min after CPET; a decrease of $\geq 10 \%$ in at least one of these time points was defined as EIB.

\section{Cardiopulmonary Exercise Testing}

Exercise testing was performed in an air-conditioned room at a temperature of $21-22^{\circ} \mathrm{C}$ and relative humidity of 50-60\%, using the Ultima CPX CPET (MGC Diagnostics, Saint Paul, MN) with a cycle ergometer. Following a 3 min warm-up period of free pedaling, the imposed workload was increased in a linear ramp pattern by 15 watts/min if subject's height was $<150 \mathrm{~cm}$ or by 20 watts/min if subject's height was $\geq 150 \mathrm{~cm}$, up to exhaustion. ${ }^{23}$ Participants were instructed to maintain a constant pedaling rate of $60 \pm 5 \mathrm{rpm}$ throughout the trial. Continuous electrocardiographic, blood pressure, and oxygen saturation $\left(\mathrm{SpO}_{2}\right.$; special ear-lobe sensor) monitoring was also undertaken. The test was terminated when the child was unable to maintain the pedaling speed despite encouragement or when specific adverse events (eg symptoms: chest pain, excessive dyspnea, dizziness, headache, nausea, pallor; electrocardiographic abnormalities; $\mathrm{SpO}_{2}<92 \%$ ) occurred. ${ }^{24}$

The following variables were recorded: workload (in watts), oxygen uptake ( $\mathrm{VO}_{2} ;$ in $\left.\mathrm{mL} / \mathrm{kg} / \mathrm{min}\right)$, carbon dioxide output $\left(\mathrm{VCO}_{2}\right.$; in $\left.\mathrm{mL} / \mathrm{kg} / \mathrm{min}\right)$, respiratory exchange ratio (RER; $\dot{\mathrm{VCO}}_{2} / \mathrm{VO}_{2}$ ), heart rate (HR; in bpm), and minute ventilation (VE; in $\mathrm{L} / \mathrm{min}$ ); all parameters were obtained in a breath-by-breath mode and averaged over an 8-breath moving window. The maximum ("peak") value of $\mathrm{VO}_{2}, \mathrm{VCO}_{2}$,
RER, VE and HR was defined as the average value of the last $15 \mathrm{~s}$ of the trial. Predicted $\mathrm{VO}_{2}$ peak values were calculated using the equations proposed by Cooper et al. ${ }^{25}$ The ratios $\dot{\mathrm{VO}}_{2}$ peak to maximum workload $\left(\dot{\mathrm{VO}}_{2}\right.$ peak/w), $\dot{\mathrm{VO}}_{2}$ peak to maximum HR ( $\dot{\mathrm{VO}}{ }_{2}$ peak/HR), VEpeak/ $\dot{\mathrm{VO}}_{2}$ peak, VEpeak/ $\dot{\mathrm{VCO}}_{2}$ peak, VEeak to maximal voluntary ventilation (MVV, calculated as $35 \times \mathrm{FEV}_{1}$ ) and VEpeak to $30 \times \mathrm{FEV}_{1}{ }^{26}$ were determined. The ventilation efficiency slope ( $\dot{\mathrm{VE}} / \dot{\mathrm{VCO}}_{2}$ slope $)$ was also computed. The anaerobic threshold (AT) was determined by the V-slope method, while the level of breathing discomfort at the end of the test was assessed by the modified 10-point Borg scale.

A CPET trial was considered maximal when: a) the child presented signs of maximum physical effort (sweating, fatigue), b) the HR was $\geq 85 \%$ of the age-predicted value, and c) the RER was $>1.05$. Ventilatory limitation was defined as maximum VEpeak $/ 30 \times \mathrm{FEV}_{1}>0.85^{26}$ in a child with $\mathrm{VO}_{2}$ peak $<80 \%$ of predicted. Participants with $\mathrm{VO}_{2}$ peak $<80 \%$ of predicted and an early plateau in the $\dot{\mathrm{VO}}_{2}$ peak/HR or a drop of $\dot{\mathrm{VO}}_{2}$ peak/HR before peak exercise were categorized as having cardiac impairment. Physical deconditioning was defined as $\mathrm{VO}_{2}$ peak $<80 \%$ of predicted, without ventilatory limitation or cardiac impairment. $^{27}$

\section{Statistics}

Continuous variables are presented as mean \pm SD or median with range; comparisons between the two study groups were performed with Student's $t$ or Mann-Whitney U-tests, respectively. The chi-square or Fisher's exact tests were used for the comparisons of categorical variables. Univariable and multivariable linear regression was used to assess the effect of sex, age, BMI (z-score), asthma, EIB, ventilatory limitation and physical deconditioning on the achieved $\mathrm{VO}_{2}$ peak. All predictors were first tested for collinearity and if it was present, the predictor was excluded from the model. Statistical analyses were performed with the IBM SPSS version 25 (IBM Corp., Armonk, NY).

\section{Results}

A total of 106 children participated in the study, 45 with controlled mild-moderate asthma and 61 healthy controls. The baseline characteristics of the study population are presented in Table 1. There were no differences in age, height, 
and weight between the two groups; children with asthma had higher BMI z-score. The median ACT score of children with asthma was 23 (range 21-25). According to the IPAQ-SF, $62.2 \%$ of the participants with asthma and $29.5 \%$ of their healthy peers were categorized as inactive $(\mathrm{P}=0.002$; Table 1$)$.

The children in the asthma group had lower $\mathrm{FEV}_{1}$, $\mathrm{FEV}_{1} / \mathrm{FVC}$ and $\mathrm{FEF}_{25-75}$ as compared with controls (Table 1). At study entry, the lowest value for $\mathrm{FEV}_{1}$ in children with asthma was $82.7 \%$ of predicted $(-1.52$ z-scores) and for $\mathrm{FEV}_{1} / \mathrm{FVC} 0.81$ ( $-0.97 \mathrm{z}$-scores). The lowest values for $\mathrm{FEV}_{1}$ and $\mathrm{FEV}_{1} / \mathrm{FVC}$ in the non-asthma group were $85.3 \%$ of predicted $(-1.23 \mathrm{z}$-scores) and 0.84 ( -0.29 z-scores), respectively.

All participants performed CPET that met the maximal criteria. The CPET parameters are presented in Table 2 . The children with asthma achieved significantly lower mean $\dot{\mathrm{VO}}_{2}$ peak, maximal work, and $\dot{\mathrm{VO}}_{2}$ peak/HR; they also had lower VEpeak, VEpeak/MVV and VEpeak $/ 30 \times \mathrm{FEV}_{1}$ (Table 2). Conversely, there were no differences between the two groups in $\mathrm{HRmax}, \mathrm{VO}_{2}$ peak /work, RER, VEpeak/V을 ${ }_{2}$ peak, $\operatorname{VEpeak} / \mathrm{VCO}_{2}$ peak, ventilation efficiency slope and level of desaturation; the Borg dyspnea scores were also comparable (Table 2).

Reduced cardiopulmonary exercise capacity $\left(\mathrm{VO}_{2}\right.$ peak $<80 \%$ of predicted) was noted in $53.3 \%$ of children with asthma and in $16.4 \%$ of controls $(\mathrm{P}<0.001)$ (Figure 1$)$. Five children, all with asthma, were diagnosed with EIB. Ventilatory limitation was noted in $13.3 \%$ of those with asthma and in $1.6 \%$ of controls $(\mathrm{P}=0.040)$; two children (4.4\% of those with asthma) had both ventilatory limitation and EIB. Physical deconditioning was noted in 37.8\% of participants with asthma and in $14.8 \%$ of controls $(\mathrm{P}=0.013)$ (Figure 1).

Children with asthma, and those with increased BMI or physical deconditioning were more likely to achieve a lower $\mathrm{VO}_{2}$ peak. However, physical deconditioning emerged as the only significant determinant of cardiopulmonary exercise capacity in the multivariable regression model, irrespective of asthma diagnosis, BMI z-score, ventilatory limitation and presence of EIB (Table 3).

\section{Discussion}

In this cross-sectional study we explored the exercise capacity of children with controlled mild-moderate asthma and the underlying mechanisms that may lead to its reduction in this population. We found that children with asthma were less exercise tolerant than their non-asthmatic peers; the main determinant of reduced exercise capacity was physical deconditioning, while the contribution of EIB and ventilatory limitation was minimal.

Studies employing CPET to assess the exercise capacity of children with asthma are sparse. Two earlier reports showed that children with newly diagnosed ${ }^{13}$ and those with persistent asthma ${ }^{14}$ had lower $\dot{\mathrm{VO}}_{2}$ peak than their healthy peers; however, the underlying mechanisms of reduced exercise capacity (ie EIB, ventilatory or cardiovascular limitation) were not explored. ${ }^{13,14}$ A recent casecontrol study confirmed that children with severe refractory asthma may be less tolerant to exercise. ${ }^{15}$ Using a CPET protocol similar to ours, the investigators also showed that EIB (documented in $30 \%$ of cases), physical deconditioning ( $25 \%$ of cases), and a combination of the two (25\% of cases) could explain the reduced exercise capacity in that cohort. ${ }^{15}$ Similar studies in adults have yield contradictory results. Earlier reports suggest that the reduced $\mathrm{VO}_{2}$ peak in patients with controlled moderate-to-severe asthma cannot be attributed to airflow limitation, ${ }^{28}$ while others have shown that the reduced exercise capacity is linked to small airways disease, especially in subjects with EIB. ${ }^{29}$ Finally, more recent reports conclude that most adults with mild-to-moderate asthma have normal $\mathrm{VO}_{2}$ peak and, thus, no truly reduced exercise capacity. ${ }^{30}$

To our knowledge, our study is the first to explore the mechanisms of reduced exercise capacity in children with well-controlled mild-to-moderate asthma. We found that the contribution of EIB was minimal; EIB was noted in $11.1 \%$ of participants with asthma and did not emerge as a significant determinant of $\mathrm{VO}_{2}$ peak. In a recent metaanalysis, the pooled prevalence of EIB among children and adolescents with asthma was $46 \%$ (95\% CI: 39-53\%), but the estimates varied significantly depending on the method of exercise challenge and the severity of the disease (17$41 \%$ in mild versus $52-99 \%$ in severe asthmatics). ${ }^{31}$ However, the analyzed studies were all based on EIBoriented protocols, ie methods of exercise challenge that differ significantly from the standard incremental CPET protocol used in our study. ${ }^{32}$ In a study methodologically comparable to ours, EIB was documented in $55 \%$ of participants with asthma and emerged as major determinant of reduced exercise capacity, but that study enrolled only subjects with severe refractory disease (ACT scores between 18 and 23). ${ }^{15}$ 
Table I Characteristics of the Study Groups

\begin{tabular}{|c|c|c|c|}
\hline & Asthma Group ( $n=45)$ & Control Group $(n=6 I)$ & P-value \\
\hline Male gender, n (\%) & $32(71.1)$ & $49(80.3)$ & 0.269 \\
\hline Age, years & $12.2 \pm 2.1$ & $12.5 \pm 2.3$ & $0.48 \mathrm{I}$ \\
\hline Height, cm & $154.9 \pm 13.8$ & $157.5 \pm 15.1$ & 0.360 \\
\hline Weight, kg & $52.0 \pm 15.5$ & $50.1 \pm 13.7$ & 0.505 \\
\hline $\mathrm{BMI}, \mathrm{kg} / \mathrm{m}^{2}$ & $21.4 \pm 4.5$ & $19.9 \pm 3.4$ & 0.056 \\
\hline BMI, z-score & $0.80 \pm 0.91$ & $0.32 \pm 1.09$ & 0.019 \\
\hline ACT, median (range) & $23(2 I-25)$ & NA & \\
\hline \multicolumn{4}{|c|}{ Physical activity questionnaire } \\
\hline Inactive, n (\%) & $28(62.2)$ & $18(29.5)$ & 0.002 \\
\hline \multicolumn{4}{|l|}{ Spirometry } \\
\hline $\mathrm{FEV}_{\mathrm{l}}, \mathrm{L}$ & $2.4 \pm 0.6$ & $2.9 \pm 0.9$ & $<0.001$ \\
\hline $\mathrm{FEV}_{1}, \%$ & $91.3 \pm 9.4$ & $100.9 \pm 10.5$ & $<0.001$ \\
\hline $\mathrm{FEV}_{\text {, z-score }}$ & $-0.74 \pm 0.81$ & $0.08 \pm 0.89$ & $<0.001$ \\
\hline FVC, L & $2.9 \pm 0.7$ & $3.3 \pm 1.1$ & 0.019 \\
\hline FVC, \% & $90.4 \pm 14.1$ & $96.4 \pm 12.2$ & 0.021 \\
\hline FVC, z-score & $-0.83 \pm 1.21$ & $-0.32 \pm 1.04$ & 0.022 \\
\hline $\mathrm{FEV}_{1} / \mathrm{FVC}$ & $0.87 \pm 0.05$ & $0.91 \pm 0.04$ & $<0.001$ \\
\hline $\mathrm{FEV}_{1} / \mathrm{FVC}$ z-score & $0.01 \pm 0.81$ & $0.81 \pm 0.83$ & $<0.001$ \\
\hline $\mathrm{FEF}_{25-75}, \mathrm{~L} / \mathrm{sec}$ & $2.1 \pm 1.1$ & $2.8 \pm 1.3$ & 0.004 \\
\hline $\mathrm{FEF}_{25-75}, \%$ & $80.3 \pm 14.9$ & $99.4 \pm 19.6$ & $<0.001$ \\
\hline $\mathrm{FEF}_{25-75}, \mathrm{z}$-score & $-0.94 \pm 0.73$ & $-0.04 \pm 0.93$ & $<0.001$ \\
\hline
\end{tabular}

Note: Values are mean \pm SD unless stated otherwise.

Abbreviations: BMI, body mass index; ACT, asthma control test; $\mathrm{FEV}_{\mathrm{l}}$, forced expiratory volume at I s; FVC, forced vital capacity; $\mathrm{FEF}_{25-75}$, forced expiratory flow at $25-$ $75 \%$ of FVC.

The effect of ventilatory limitation in our cohort, was also small; only $13.3 \%$ of children with asthma had a VEpeak $/ 30 \times \mathrm{FEV}_{1} \geq 0.85,{ }^{26}$ yet without significant influence on the achieved $\dot{\mathrm{VO}}_{2}$ peak. The only other report that has examined ventilatory limitation in children with asthma using CPET showed, surprisingly, that this mechanism was absent in patients with severe refractory disease. ${ }^{15}$ The different definition for ventilation limitation (ie $\operatorname{VEpeak} / \mathrm{MVV} \geq 0.80$ ) compared to VEpeak $/ 30 \times \mathrm{FEV}_{1} \geq 0.85$ in our study, may account for this striking difference. Of note, recent evidence suggests that the criterion VEpeak $/ 30 \times \mathrm{FEV}_{1} \geq 0.85$ is significantly more accurate than the classical VEpeak/MVV $\geq 0.80$ approach in children. ${ }^{26}$ Interestingly, in $4.4 \%$ $(\mathrm{N}=2)$ of participants with asthma both ventilatory limitation and EIB were documented. This finding most likely confirms the presence of "breakthrough" EIB (ie bronchoconstriction that occurs during the exercise) ${ }^{12}$ in this population. The small number of children in this group did not permit for further analysis of this intriguing finding. It should be noted that all participants had a normal echocardiographic examination on the day of enrollment, and none presented signs of cardiovascular impairment during CPET.

The most important finding of this study is that physical deconditioning, defined as reduced cardiopulmonary exercise capacity in the absence of ventilatory limitation 
Table 2 CPET Parameters

\begin{tabular}{|c|c|c|c|}
\hline & Asthma Group ( $n=45)$ & Control Group $(n=61)$ & P-value \\
\hline Maximal work, watts & $136.3 \pm 29.2$ & $151.9 \pm 39.0$ & 0.021 \\
\hline Test time, $\min$ & $10.1 \pm 1.4$ & $10.6 \pm 1.7$ & 0.075 \\
\hline $\mathrm{VO}_{2}$ peak, $\mathrm{mL} / \mathrm{kg} / \mathrm{min}$ & $36.1 \pm 7.4$ & $41.3 \pm 6.7$ & $<0.001$ \\
\hline $\mathrm{VO}_{2}$ peak, \% predicted & $85.2 \pm 19$ & $93.8 \pm 16.5$ & 0.015 \\
\hline $\mathrm{VO}_{2}$ at $\mathrm{AT}, \mathrm{mL} / \mathrm{kg} / \mathrm{min}$ & $25.1 \pm 6.6$ & $29.0 \pm 6.0$ & 0.002 \\
\hline AT $\%$ VंO ${ }_{2}$ peak, $\%$ & $69.1 \pm 9.5$ & $70.6 \pm 10.4$ & 0.448 \\
\hline HRmax, bpm & $188 \pm 9$ & $188 \pm 10$ & 0.917 \\
\hline $\mathrm{VO}_{2}$ peak/HR, mL/beat & $9.6 \pm 2.3$ & $10.9 \pm 3.1$ & 0.020 \\
\hline $\mathrm{VO}_{2}$ peak/work, $\mathrm{mL} /$ watts $\times$ min & $13.2 \pm 1.2$ & $13.4 \pm 1.5$ & 0.478 \\
\hline RER & $1.12(I . I-1.4)$ & $1.16(1.1-1.4)$ & 0.066 \\
\hline VE, L/min & $65.1 \pm 14.7$ & $72.8 \pm 19.4$ & 0.023 \\
\hline$\dot{\mathrm{VE}} / \mathrm{MVV}$ & $0.79 \pm 0.12$ & $0.73 \pm 0.14$ & 0.045 \\
\hline $\mathrm{VE} / 30 \times \mathrm{FEV}$ & $0.77 \pm 0.12$ & $0.71 \pm 0.13$ & 0.041 \\
\hline$\dot{\mathrm{VE}} / \mathrm{VOO}_{2}$ & $36.4 \pm 4.4$ & $36.1 \pm 4.7$ & 0.767 \\
\hline$\dot{\mathrm{VE}} / \mathrm{VCO}_{2}$ & $31.2 \pm 3.3$ & $31.6 \pm 3.4$ & 0.526 \\
\hline Ventilation efficiency slope & $29.6 \pm 3.4$ & $30.2 \pm 3.5$ & 0.437 \\
\hline $\mathrm{SpO}_{2}$ decrease & $2(I-3)$ & $2(I-3)$ & 0.876 \\
\hline Minimum $\mathrm{SpO}_{2}$ & 95 (94-98) & 96 (94-98) & 0.304 \\
\hline Borg dyspnea & $7(5-8)$ & $7(6-8)$ & 0.422 \\
\hline
\end{tabular}

Notes: Values are mean \pm SD or median (range) and were compared with $t$ test or Mann-Whitney $U$, respectively.

Abbreviations: CPET, cardiopulmonary exercise testing; AT, anaerobic threshold; HR, heart rate; RER, respiratory exchange ratio; MVV, maximal voluntary ventilation.

or cardiac impairment, was documented in $37.8 \%$ of children with asthma and in $70.8 \%$ of children with asthma and low $\mathrm{VO}_{2}$ peak. Furthermore, physical deconditioning emerged as the only significant determinant of $\mathrm{VO}_{2}$ peak in our cohort, irrespective of asthma diagnosis and the presence of EIB or ventilatory limitation. Physical deconditioning also outperformed and masked the effect of BMI, which has been associated with reduced exercise capacity in other studies. ${ }^{8,11}$ The above finding is of particular importance, because it suggests that the decreased aerobic fitness in children with asthma ${ }^{7-15}$ should not be attributed to underlying cardio-respiratory mechanisms that reduce their exercise capacity, but rather to the lack of regular physical activity that leads to deconditioning. Indeed, $60.7 \%$ of our children with asthma who were categorized as inactive were also physically deconditioned, while all children with asthma and physical deconditioning were inactive. To our knowledge, only one other study has explored the underlying mechanisms of reduced exercise capacity in children with asthma by means of CPET; ${ }^{15}$ that study focused on subjects with severe refractory asthma and reached conclusions similar to ours regarding the contribution of physical deconditioning. Of note, maximal incremental CPET has been shown to be the only reliable method to systematically assess the underlying etiology of reduced exercise capacity. ${ }^{33}$

The relationship between asthma and physical activity in childhood remains unclear. A meta-analysis of studies that have used accelerometers concluded that children with asthma are engaged in similar amount of 


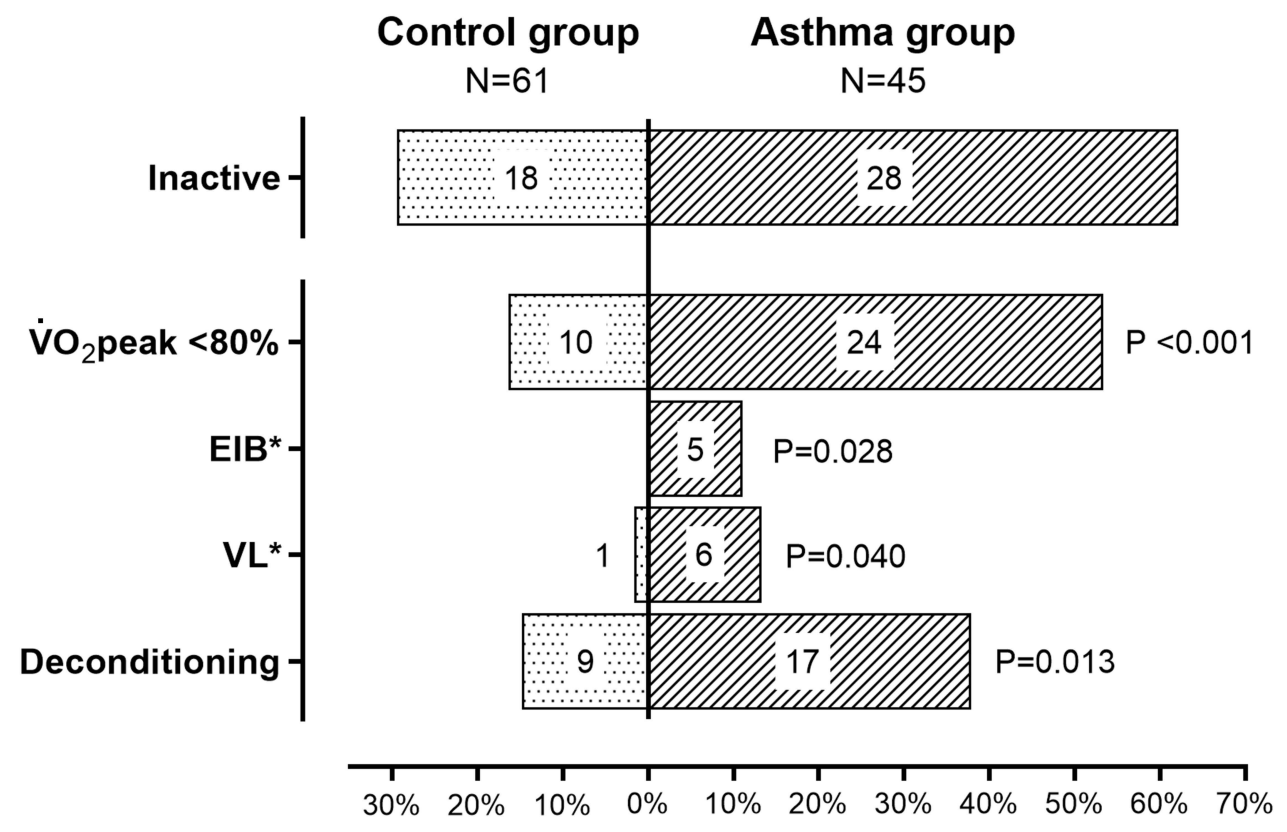

Figure I Numbers (in boxes) and percentages (x-axis) of children with low $\mathrm{VO}_{2}$ peak, EIB, $\mathrm{VL}$ and physical deconditioning in the two study groups. The number of inactive participants is also presented. Comparisons were performed with chi-square or Fisher's exact test. *Two children had both ElB and VL.

Abbreviations: EIB, exercise-induced bronchoconstriction; VL, ventilatory limitation.

physical activity as their healthy peers; ${ }^{7}$ subsequent studies have also confirmed these findings. ${ }^{9,11}$ On the other hand, children with exercise induced bronchoconstriction, ${ }^{11,12}$ and those with newly diagnosed, ${ }^{10}$ uncontrolled, ${ }^{10,13}$ or severe asthma ${ }^{13}$ have been found to be less active than their peers. Since our study included children and adolescents with controlled mild-to-moderate asthma, the observed differences in physical activity between cases and controls were unexpected. A likely explanation is that the children of our cohort who were less active reported realistic estimates regarding the frequency and duration of their physical activities, as they were aware that an objective measure of cardiovascular fitness (ie the CPET) would follow.

Table 3 Determinants of Cardiopulmonary Exercise Performance*

\begin{tabular}{|l|l|l|l|l|}
\hline \multirow{2}{*}{ Factor } & \multicolumn{2}{l|}{ Unadjusted Effect } & \multicolumn{2}{l|}{$\begin{array}{l}\text { Adjusted Effect } \\
R^{2} 0.455\end{array}$} \\
\cline { 2 - 5 } & Beta & $\mathbf{P}$ & Beta \\
\hline Male gender & $0.089(-0.1 \mathrm{I}$ to 0.28$)$ & 0.367 & $0.044(-0.20$ to $0.1 \mathrm{I})$ & 0.567 \\
\hline Age & $0.143(-0.05$ to 0.34$)$ & 0.143 & $0.005(-0.15$ to 0.16$)$ & 0.949 \\
\hline BMI, z-score & $-0.247(-0.44$ to -0.06$)$ & 0.011 & $-0.113(-0.28$ to 0.05$)$ & 0.173 \\
\hline Asthma & $-0.236(-0.42$ to -0.05$)$ & 0.015 & $-0.007(-0.16$ to 0.17$)$ & 0.932 \\
\hline ElB & $-0.025(-0.22$ to 0.17$)$ & 0.801 & $-0.057(-0.22$ to 0.11$)$ & 0.497 \\
\hline Ventilatory limitation & $-0.018(-0.21$ to 0.17$)$ & 0.853 & $-0.108(-0.28$ to 0.06$)$ & 0.213 \\
\hline Physical deconditioning & $-0.645(-0.79$ to 0.50$)$ & $<0.001$ & $-0.653(-0.82$ to 0.49$)$ & $<0.001$ \\
\hline
\end{tabular}

Notes: Values are standardized linear regression coefficients beta with $95 \%$ confidence intervals in parenthesis. The unadjusted effect (univariable models) refers to the effect of each factor separately. The adjusted effect (multivariable model) refers to the combined effect of these factors when adjusted for each other. The achieved power of the multivariable model was $99.99 \%$. $* \mathrm{VO}_{2}$ peak \% predicted.

Abbreviations: BMI, body mass index; EIB, exercise-induced bronchoconstriction. 
The fact that the IPAQ-SF classification (ie active versus inactive) was in accordance with the percentage of children categorized as fit or unfit by CPET analysis (Figure 1), renders further support to the above hypothesis and to the validity of our results.

Physical activity is of great importance for children because it promotes cardiovascular health, improves cognitive function, and establishes the foundations for lifelong health and well-being. ${ }^{19,20}$ Patients with asthma may have additional benefits, since exercise training reduces airway inflammation and bronchial hyperresponsiveness, thus improving respiratory symptoms and quality of life. ${ }^{34}$ Moreover, the physically active children with asthma have lower body fat and are less likely to develop obesity. ${ }^{35}$ Unfortunately, despite these benefits, imposed exercise restrictions are not uncommon in this population, ${ }^{4}$ and relate primarily to parental and/or children's perception that more strenuous activities may precipitate asthma symptoms and affect physical performance, thus leading to devaluation by the social environment. ${ }^{6}$ In this regard, the findings of the present study are of importance, since we show that children with mild-to-moderate asthma are less tolerant to exercise due to physical deconditioning and not because of underlying cardio-respiratory mechanisms which limit their exercise capacity. Although the cross-sectional design of our study does not permit us to fully explore the direction of causality, the assumption that children with asthma may have adopted a less active lifestyle emerges as more plausible; such an indolent lifestyle may progressively lead to physical deconditioning and further deterioration of their exercise capacity which may, in turn, feed into a vicious cycle. Thus, regular physical activity, such as training and participation in sports, should be encouraged in all children with mild-to-moderate asthma. The importance of a thorough evaluation of the exercise capacity in these children, ideally by CPET, should also be highlighted.

Inevitably, our study has some limitations. First, the level of physical activity was not assessed by objective tools (eg accelerometers). The IPAQ-SF is -in principle- a subjective method; however, a systematic analysis of physical activity was not within the purposes of the present study. Second, measurements of allergic airway inflammation were not employed and, thus, it is not possible to assess its possible contribution. However, the study was performed in the summer months when the weather in Greece is typically hot and dry and the prevalence of respiratory allergies is extremely low. Moreover, as already discussed, the standard incremental CPET protocol may not be appropriate to assess the presence of EIB. ${ }^{32}$ Nevertheless, in contrast to similar studies, ${ }^{15}$ our cohort consisted of children with well-controlled mild-to-moderate asthma; therefore, the low prevalence of EIB reported herein is most likely valid. Finally, this study was not designed to assess the underlying causes that may lead children with mild-to-moderate asthma to inactivity and physical deconditioning. These children may be reluctant to participate in physical activities due to the belief that the disease has affected their performance or that exercise may precipitate asthma symptoms, thus leading to stigmatization by their social environment. Parents, on the other hand, may not encourage children with asthma to participate in physical activities due to the misunderstanding that exercise may be harmful or due to lack of appropriate specialist guidance. Future studies should focus on these important issues, in order to uncover possible targets for individual- and familyoriented management strategies.

\section{Conclusions}

Children and adolescents with controlled mild-to-moderate asthma seem to be less capable of strenuous exercise than their healthy peers. However, the decreased exercise capacity in this population is mainly attributed to physical deconditioning, while the contribution of ventilatory limitation and/or EIB is minimal. Therefore, pediatric patients with mild-to-moderate asthma should be encouraged to participate in physical activities systematically and without constraints.

\section{Abbreviations}

CPET, cardiopulmonary exercise testing; EIB, exerciseinduced bronchoconstriction; ACT, asthma control test; IPAQ-SF, international physical activity questionnaire short form.

\section{Data-Sharing Statement}

Data supporting the findings of this manuscript may be available from the corresponding author on reasonable request.

\section{Acknowledgments}

We would like to thank all participating children and their families for their enthusiastic participation in this study. 


\section{Author Contributions}

All authors made a significant contribution to the work reported, whether that is in the conception, study design, execution, acquisition of data, analysis and interpretation, or in all these areas; took part in drafting, revising or critically reviewing the article; gave final approval of the version to be published; have agreed on the journal to which the article has been submitted; and agree to be accountable for all aspects of the work.

\section{Funding}

This research is financed by the Hellenic Foundation for Research and Innovation (H.F.R.I.) through the "1st Call for H.F.R.I. Scholarships to $\mathrm{PhD}$ Candidates" to promote actions that support new scientists. Scholarship Recipient: Dr. Olga Lagiou (\# 129322/I2). The publication of this paper was financed by the University of Patras.

\section{Disclosure}

The authors declare no conflicts of interest in relation to the present work.

\section{References}

1. Global Asthma Network. The global asthma report; 2018. Available from: www.globalasthmareport.org. Accessed August 16, 2021.

2. Global Initiative for Asthma. Global Initiative for Asthma: global strategy for asthma management and prevention (Updated 2021); 2020 Available from: www.ginasthma.org. Accessed August 16, 2021.

3. Hughes D. Childhood asthma and exercise. Paediatr Child Health. 2014;19(9):467-468. doi:10.1093/pch/19.9.467

4. Lang DM, Butz AM, Duggan AK, Serwint JR. Physical activity in urban school-aged children with asthma. Pediatrics. 2004;113(4): e341-346. doi:10.1542/peds.113.4.e341

5. Dantas FM, Correia MA, Silva AR, Peixoto DM, Sarinho ES, Rizzo JA. Mothers impose physical activity restrictions on their asthmatic children and adolescents: an analytical cross-sectional study. BMC Public Health. 2014;14(1):287-294. doi:10.1186/14712458-14-287

6. Williams B, Powell A, Hoskins G, Neville R. Exploring and explaining low participation in physical activity among children and young people with asthma: a review. BMC Fam Pract. 2008;9(1):40-51. doi:10.1186/1471-2296-9-40

7. Cassim R, Koplin JJ, Dharmage SC, et al. The difference in amount of physical activity performed by children with and without asthma: a systematic review and meta-analysis. $J$ Asthma. 2016;53 (9):882-892. doi:10.1080/02770903.2016.1175474

8. Sousa AW, Cabral AL, Martins MA, Carvalho CR. Daily physical activity in asthmatic children with distinct severities. J Asthma 2014;51(5):493-497. doi:10.3109/02770903.2014.888571

9. Matsunaga NY, Oliveira MS, Morcillo AM, Ribeiro JD, Ribeiro MAGO, Toro AADC. Physical activity and asthma control level in children and adolescents. Respirology. 2017;22 (8):1643-1648. doi:10.1111/resp.13093

10. Pike KC, Griffiths LJ, Dezateux C, Pearce A. Physical activity among children with asthma: cross-sectional analysis in the UK millennium cohort. Pediatr Pulmonol. 2019;54(7):962-969. doi:10.1002/ppul.24314
11. Anthracopoulos MB, Fouzas S, Papadopoulos M, et al. Physical activity and exercise-induced bronchoconstriction in Greek schoolchildren. Pediatr Pulmonol. 2012;47(11):1080-1087. doi:10.1002/ppul.22620

12. van der Kamp MR, Thio BJ, Tabak M, Hermens HJ, Driessen J, van der Palen J. Does exercise-induced bronchoconstriction affect physical activity patterns in asthmatic children? $J$ Child Health Care. 2020;24(4):577-588. doi:10.1177/1367493519881257

13. Vahlkvist S, Pedersen S. Fitness, daily activity and body composition in children with newly diagnosed, untreated asthma. Allergy. 2009;64 (11):1649-1655. doi:10.1111/j.1398-9995.2009.02081.x

14. Villa F, Castro AP, Pastorino AC, et al. Aerobic capacity and skeletal muscle function in children with asthma. Arch Dis Child. 2011;96 (6):554-559. doi:10.1136/adc.2011.212431

15. Faleiro RC, Mancuzo EV, Lanza FC, et al. Exercise limitation in children and adolescents with severe refractory asthma: a lack of asthma control? Front Physiol. 2021;11:620736. doi:10.3389/ fphys.2020.620736

16. Frima ES, Theodorakopoulos I, Gidaris D, et al. Lung function variability in children and adolescents with and without asthma (LUV Study): protocol for a prospective, nonrandomized, clinical trial. JMIR Res Protoc. 2020;9(8):e20350. doi:10.2196/20350

17. Cole TJ, Lobstein T. Extended international (IOTF) body mass index cut-offs for thinness, overweight and obesity. Pediatr Obes. 2012;7 (4):284-294. doi:10.1111/j.2047-6310.2012.00064.x

18. Papathanasiou G, Georgoudis G, Papandreou M, et al. Reliability measures of the short International Physical Activity Questionnaire (IPAQ) in Greek young adults. Hellenic $J$ Cardiol. 2009;50 (4):283-294.

19. Piercy KL, Troiano RP, Ballard RM, et al. Physical Activity Guidelines for Americans. 2nd ed. Washington DC: U.S. Department of Health and Human Services; 2018: 46-54

20. NHS. Physical activity guidelines for children and young people; 2019. Available from: https://www.nhs.uk/live-well/exercise/physi cal-activity-guidelines-children-and-young-people/. Assessed August $16,2021$.

21. Miller MR, Hankinson J, Brusasco V, et al. ATS/ERS Task Force. Standardisation of spirometry. Eur Respir J. 2005;26(2):319-338. doi:10.1183/09031936.05.00034805

22. Quanjer PH, Stanojevic S, Cole TJ, Baur X, Hall GL, Culver BH. ERS Global Lung Function Initiative. Multi-ethnic reference values for spirometry for the 3-95-yr age range: the global lung function 2012 equations. Eur Respir J. 2012;40(6):1324-1343. doi:10.1183/ 09031936.00080312

23. Godfrey S, Davies CT, Wozniak E, Barnes CA. Cardio-respiratory response to exercise in normal children. Clin Sci. 1971;40 (5):419-431. doi:10.1042/cs0400419

24. Paridon SM, Alpert BS, Boas SR, et al; American Heart Association Council on Cardiovascular Disease in the Young, Committee on Atherosclerosis, Hypertension, and Obesity in Youth. Clinical stress testing in the pediatric age group: a statement from the American Heart Association Council on Cardiovascular Disease in the Young, Committee on Atherosclerosis, Hypertension, and Obesity in Youth. Circulation. 2006;113(15):1905-1920. doi:10.1161/CIRCULA TIONAHA.106.174375

25. Cooper DM, Weiler-Ravell D, Whipp BJ, Wasserman K. Aerobic parameters of exercise as a function of body size during growth in children. J Appl Physiol Respir Environ Exerc Physiol. 1984;56 (3):628-634. doi:10.1152/jappl.1984.56.3.628

26. Pianosi PT, Smith JR. Ventilatory limitation of exercise in pediatric subjects evaluated for exertional dyspnea. Front Physiol. 2019;10:20. doi:10.3389/fphys.2019.00020

27. American Thoracic Society; American College of Chest Physicians. ATS/ACCP Statement on cardiopulmonary exercise testing. Am $J$ Respir Crit Care Med. 2003;167(2):211-277. doi:10.1164/ rccm.167.2.211 
28. Clark CJ, Cochrane LM. Assessment of work performance in asthma for determination of cardiorespiratory fitness and training capacity. Thorax. 1988;43:745-749. doi:10.1136/thx.43.10.745

29. Chryssanthopoulos C, Maksud MG, Funahashi A, Hoffmann RG, Barboriak JJ. An assessment of cardiorespiratory adjustments of asthmatic adults to exercise. $J$ Allergy Clin Immunol. 1979;63:321-327. doi:10.1016/0091-6749(79)90126-X

30. Garfinkel SK, Kesten S, Chapman KR, Rebuck AS. Physiologic and nonphysiologic determinants of aerobic fitness in mild to moderate asthma. Am Rev Respir Dis. 1992;145:741-745. doi:10.1164/ajrccm/ 145.4 Pt 1.741

31. de Aguiar KB, Anzolin M, Zhang L. Global prevalence of exercise-induced bronchoconstriction in childhood: a meta-analysis. Pediatr Pulmonol. 2018;53(4):412-425. doi:10.1002/ppul.23951
32. Stensrud T, Carlsen KH. Can one single test protocol for provoking exercise-induced bronchoconstriction also be used for assessing aerobic capacity? Clin Respir J. 2008;2(1):47-53. doi:10.1111/j.1752699X.2007.00030.x

33. Goddard T, Sonnappa S. The role of cardiopulmonary exercise testing in evaluating children with exercise induced dyspnoea. Paediatr Respir Rev. 2021;38:24-32. doi:10.1016/j.prrv.2020.08.002

34. Eichenberger PA, Diener SN, Kofmehl R, Spengler CM. Effects of exercise training on airway hyperreactivity in asthma: a systematic review and meta-analysis. Sports Med. 2013;43(11):1157-1170. doi:10.1007/s40279-013-0077-2

35. Lu KD, Manoukian K, Radom-Aizik S, Cooper DM, Galant SP. Obesity, asthma, and exercise in child and adolescent health. Pediatr Exerc Sci. 2016;28(2):264-274. doi:10.1123/pes.2015-0122

\section{Publish your work in this journal}

The Journal of Asthma and Allergy is an international, peer-reviewed open-access journal publishing original research, reports, editorials and commentaries on the following topics: Asthma; Pulmonary physiology; Asthma related clinical health; Clinical immunology and the immunological basis of disease; Pharmacological interventions and new therapies. The manuscript management system is completely online and includes a very quick and fair peer-review system, which is all easy to use. Visit http://www.dovepress.com/testimonials.php to read real quotes from published authors. 